\title{
Novel flexible light diffuser and irradiation properties for photodynamic therapy
}

\author{
Baerbel Selm \\ Markus Rothmaier \\ Martin Camenzind \\ Empa \\ Swiss Federal Laboratories for Materials Testing \\ and Research \\ Laboratory for Protection and Physiology \\ $\mathrm{CH}-9014$ St. Gallen \\ Switzerland
}

\section{Tania Khan}

Heinrich Walt

University Hospital Zürich

Research Division of Gynecology

$\mathrm{CH}-8091$ Zürich, Switzerland

\begin{abstract}
Many current light diffusers for photodynamic therapy are inflexible, and the applied light dose is difficult to adjust during treatment, especially on complex body surfaces. A thin and flexible luminous textile is developed using plastic optical fibers as a light distributor. The textile diffuser is evaluated for flexibility, irradiance, brightness distribution, and temperature rise with a 652-nm laser set to $100 \mathrm{~mW}$. The bending force of the textile diffuser resembles a defined optical film. On the textile surface, an average output power of $3.6 \pm 0.6 \mathrm{~mW} / \mathrm{cm}^{2}$ is measured, corresponding to a transmission rate of $40 \pm 3.8 \%$ on an area of $11 \mathrm{~cm}^{2}$. Aluminum backing enhances the irradiance to the face (treatment side). The measured brightness distribution seems to lie within a range similar to other photodynamic therapy (PDT) devices. A power setting of $100 \mathrm{~mW}$ increases the temperature of the textile diffuser surface of up to $27^{\circ} \mathrm{C}$, and $1 \mathrm{~W}$ raises the temperature above $40^{\circ} \mathrm{C}$. Results confirm that the flexible textile diffuser supplies suitable radiation for low fluence rate photodynamic therapy on an area of several $\mathrm{cm}^{2}$. $\odot 2007$ Society of Photo-Optical Instrumentation Engineers. [DOI: 10.1117/1.2749737]
\end{abstract}

Keywords: embroidery; light conducting; plastic optical fiber.

Paper 06352R received Dec. 1, 2006; revised manuscript received Feb. 21, 2007; accepted for publication Feb. 22, 2007; published online Jun. 22, 2007.

\section{Introduction}

Photodynamic therapy (PDT) is based on photochemical reactions involving photosensitizer molecules (e.g., porphyrin derivatives) to treat cancerous and noncancerous target tissue. $^{1-5}$ Transported by lipoproteins after intravenous injection, photosensitizer molecules accumulate primarily in fastgrowing tissues-most likely cancer cells-and after a specific interval; they are activated by a light dose of a specific wavelength. The activated photosensitizer interacts with local oxygen, creating a highly reactive singlet oxygen form, which is considered to be the main cause of cell death. ${ }^{6}$ Conventional light delivery systems consist of a nonthermal light source and a light diffuser, which is responsible for homogeneous illumination of the target cell area. Many types of light diffusers have been developed recently to address the need for topical or body cavity applications. For topical use, suitable reflectors can be used to obtain a homogeneous light distribution using a noncoherent light source ${ }^{7}$; for spot irradiations, optical fibers with microlenses have been described in the literature. ${ }^{8}$ Applications to deliver light inside body cavities have led to a multiplicity of different illuminator and diffuser devices and techniques, dependent on size, geometry, and accessibility of the solid or hollow organ to be treated. Frontal illuminators (microlens, spherical tip fiber), and cylindrical and balloon diffusers of virtually any shape are presented in the recent literature. ${ }^{9-15}$

Address all correspondence to Baerbel Selm, Empa, Swiss Federal Laboratories for Materials Testing and Research, Lerchenfeldstrasse 5, CH-9014 St. Gallen, Switzerland; Tel.: +41 71274 7153; Fax: +41 71274 7762; E-mail: baerbel.selm@empa.ch
It is well known that successful and safe PDT depends mainly on correct irradiance, ${ }^{16,17}$ as well as control of local photosensitizer concentration, tissue oxygenation, and depth of light penetration into the tissue. The disadvantage of many diffusers mentioned previously is their limited ability to adjust to complex surfaces. They are relatively inflexible or even rigid, and the applied light dose is difficult to predict and control during treatment due to insufficient distance control and unintentional movements. The main goal of this work was to produce a 2-D laminar diffuser with superior flexibility for complex superficial, intraluminal, and interstitial PDT applications. We investigated the construction and performance of this novel light diffuser, which is based on a thin textile support populated with a plurality of plastic optical fibers (POF) delivering the necessary light dose. ${ }^{18}$ Combinations of textiles with optical fibers already exist for a variety of functions in fashion design, security devices, sensorics, data communication, or automotive applications. ${ }^{19-23}$ In most cases, woven textile structures that incorporate POF are utilized. For our design we used two-thread shuttle embroidery technology for the POF instead of weaving (see Fig. 1). Light emittance was achieved by macrobending loops ${ }^{24,25}$ in the optical fibers automatically through the embroidery process during diffuser production (there is no additional step to damage the cladding in certain locations through mechanical abrasion or chemical solvents). The advantages of embroidery are numerous: 1 . POF patterns of every character (single spots, lines, curves, zigzag) can be stitched onto many substrate materials (e.g.,

1083-3668/2007/12(3)/034024/7/\$25.00 @ 2007 SPIE 


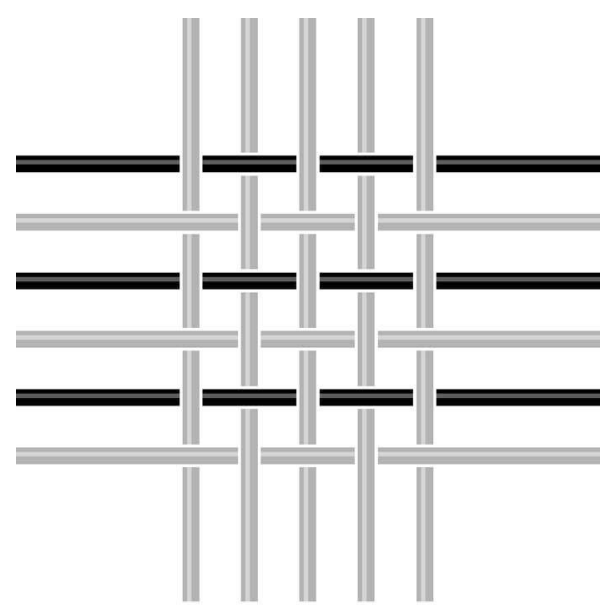

(a)

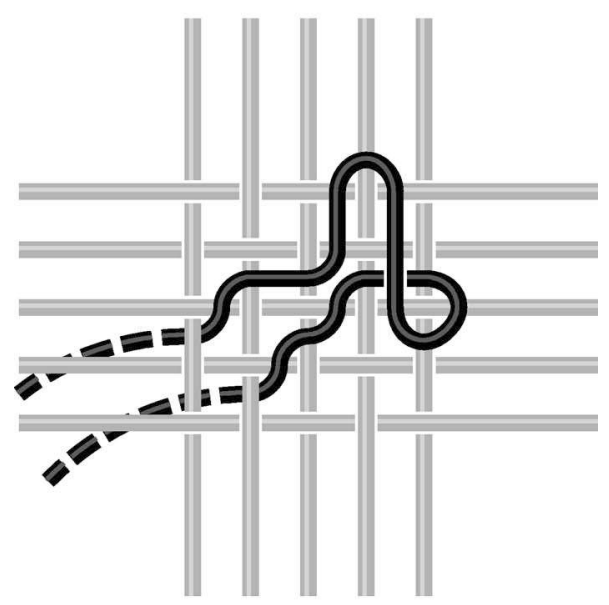

(b)

Fig. 1 Schematics of textile fabrics with integrated POF (shown in black): (a) woven structure with woven POF, and (b) woven structure as a substrate with embroidered POF (equivalent to one luminous fiber element).

textile, nonwoven, membrane, film, paper); 2. embroidery allows manufacturing of every 2-D form that adjusts easily even to complex organ or skin topologies; 3. 3-D structures can be produced; 4 . light delivery can be positioned according to diffuser design; 5. a flat textile requires significantly less space; and 6. safety is enhanced.

In this work we summarize our measurements of flexibility, radiance, and temperature rise for textile-based diffusers. The results suggest that PDT treatment by means of luminous textiles is feasible, and therefore offers an alternative light delivery with respect to common diffusers, especially for complex surfaces and body cavities.

\section{Materials and Methods}

\subsection{Design of the Flexible Light Diffuser}

The embroidery-based textile light diffusers investigated were from a single production batch (production series 3, delivered by Bischoff Textil, Saint Gallen, Switzerland), and consisted of a dense woven substrate in which plastic optical fibers were fixed using conventional yarn. The bare woven substrate con- sisted of $100 \%$ polyester multifilament with a textile finish, with a weight of $50 \mathrm{~g} / \mathrm{m}^{2}$ and 29 threads per centimeter in warp and weft (Swisstulle, Münchwilen, Switzerland). Very thin step index-plastic optical fibers (SI-POF, Poly Optical, Irvine, California) with a fiber diameter of $175 \mu \mathrm{m}$, made of polymethyl methacrylate (PMMA) (core) and a cladding of fluorinated derivatives of PMMA, were fixed with a $100 \%$ polyester twisted staple fiber yarn (7 tex x 2, Bobinerie Weishaupt, Rorschach, Switzerland). The diffusers were produced with the maximum achievable density with a stitch-tostitch distance of $1 \mathrm{~mm}$. During the regular stitching of the pattern of a fiber element, the optical fiber formed random bends and loops, and was immediately fixed on the textile surface by shuttle threads. The embroidery technique forms each optical fiber into a luminous element, using eight stitches, and cuts the shuttle threads. On the front of the woven substrate, the optical fibers form an average of 28 loops per $\mathrm{cm}^{2}$ with a loop diameter of several $100 \mu \mathrm{m}$. The distance to the proximate luminous element is $1 \mathrm{~mm}$. Both ends of the optical fiber were coupled to the light source. All 178 optical fiber ends of each diffuser were bound within a metallic coupling element with a bundle diameter of $2.7 \mathrm{~mm}$. The complete diffuser (connector, free-lying POF, and textile) had a length of $10 \mathrm{~cm}$. This textile construction resulted in a flexible diffuser that was about $2 \mathrm{~mm}$ in height and delivered a round luminous area of about $11 \mathrm{~cm}^{2}$. To improve the forward radiation characteristics (tissue interface) of the textile diffuser, a commercial reflecting aluminum foil was applied to the back to utilize the radiation from both sides of the diffuser, and to protect the patient and clinical personnel from radiation during treatment.

\subsection{Flexibility Measurements}

Because of the design of the fixed POF on the woven substrate (i.e., the POF pattern), the diffuser's flexible textile character differs lengthwise and crosswise. The forces to bend the samples in both directions through angles of 5 and $15 \mathrm{deg}$ were determined with a home-built flexural stiffness measuring instrument to estimate this influence of the POF pattern. To measure the flexibility, we compared the textile diffuser with a well-known flexible optical film (Optical Lighting Film, OLF, 2301, thickness $0.5 \mathrm{~mm}$, 3M, Rüschlikon, Switzerland), which is regular in each direction, using an experimental measurement standard of the paper industry (DIN 53121:1996-12). The center of each of the three films and the three textile diffuser samples was bent, lengthwise and crosswise, to the front and back, with a bending length of $1 \mathrm{~cm}$.

\subsection{Radiation Measurement Setup}

A sketch of the light delivery system is shown in Fig. 2. For all measurements, we used a $2-\mathrm{W}$ diode laser, wavelength $\lambda$ $=652 \mathrm{~nm}$ (Ceram Optec/Biolitec, Bonn/Jena, Germany). The emitted light was coupled into the 178-fiber bundle of the textile diffuser using a frontal light distributor (Medlight, Ecublens, Switzerland). For all measurements, a power output of $100 \mathrm{~mW}$ was adjusted at the distal frontal light distributor end as measured by a calibrated radiometric detector head (sensing area $1 \mathrm{~cm}^{2}$, Gigahertz-Optik, Puchheim, Germany), assembled at the calibrated integrating sphere $(150 \mathrm{~mm}$ in 


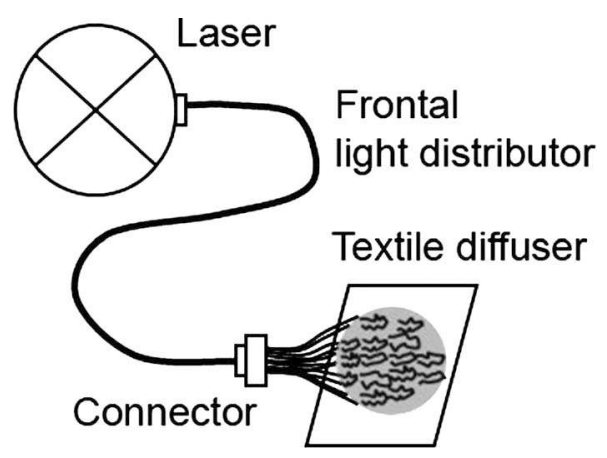

Fig. 2 Schematic diagram of the light delivery system for irradiation measurements consisting of laser, frontal light distributor, connector, and textile diffuser, including an approximately circular array of luminous fiber elements.

diameter, Gigahertz-Optik). All experiments were executed under low-light conditions in free space, in a room climate of $22^{\circ} \mathrm{C}$ and $50 \%$ relative humidity.

\subsection{Irradiance Measurements}

For the determination of the absolute radiation emission of seven textile diffusers, a round $1-\mathrm{cm}^{2}$ opening of an integrating sphere was placed in close contact with the flat textile diffuser, covering 3 to 5 embroidered luminous elements, which corresponded to about 28 optical fiber loops on the dense woven substrate. The irradiance was measured twice at each of nine different points on the diffuser. The measured values were recorded with relevant software from GigahertzOptik. The influence of the aluminum film on the irradiation was also measured and compared with a black paper backing using five measurement points on two textile diffusers.

\subsection{Brightness Distribution Measurements}

We evaluated the homogeneity of six luminous textiles, by analyzing the brightness distribution of images acquired with a charge-coupled device (CCD) camera system (3CCDCamera, 8-bit resolution, 750 lines, 380,000 pixels effective, DXC-905P Power HAD, Sony Electronics, Carl Zeiss, Zürich, Switzerland) with a suitable objective (60-fold, Konica Minolta, Hausamann Kreutzer, Saint Gallen, Switzerland). To avoid saturation, we adjusted the camera aperture and used a 0.5 filter (Kodak Wratten Gelatin Filter number 96, Kodak, Lausanne, Switzerland). The lens of the camera was at a perpendicular distance of $42 \mathrm{~cm}$ from the flat fixed textile, with the depth of focus on the textile surface.

The red channel was used and the histogram of the brightness intensity distribution of the pixels was evaluated using MATLAB ${ }^{\circledR}$ (Version 7.0, The MathWorks GmbH, Gümligen, Switzerland). The surrounding nonilluminated textile was excluded using a single template with a hole segment for all images (circular center area of $707 \mathrm{~mm}^{2}, 332$ pixels $/ \mathrm{mm}^{2}$, excluding $41.1 \%$ of the photograph). Assuming a linear correlation of brightness scale to power density, we characterized the homogeneity of the radiation using the intensity level, median (Q50), 95\% quantile (Q95), and full-width at halfmaximum (FWHM) of the brightness intensity distribution.

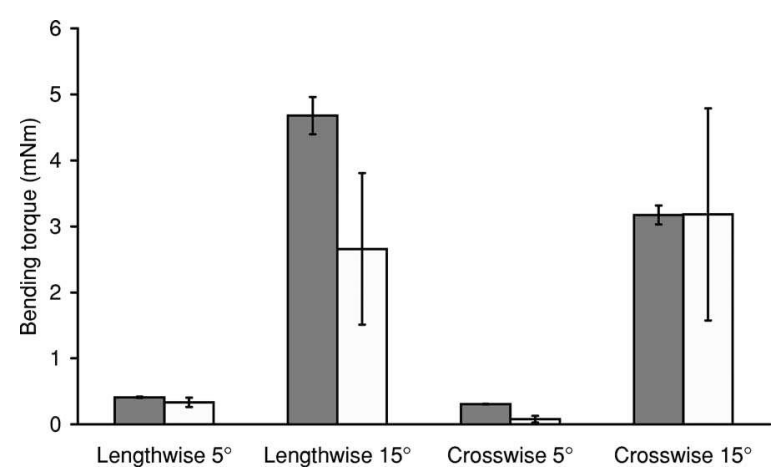

Fig. 3 Measured bending torque of the indicated systems at the given angles (white is the textile diffuser, black is the film).

\subsection{Temperature Measurements}

To gain more information about the influence of higher light fluxes on the local temperature, one light diffuser without aluminum foil was monitored at different power settings on the laser, connected as shown in Fig. 2. The temperature rise on the textile diffuser and on the connector (metal cylinder with maximum diameter of $28 \mathrm{~mm}$ and a weight of 27 gram) was measured with a thermographic camera (Agema 570, resolution $320 \times 240$ pixel, thermal accuracy $0.1 \mathrm{~K}$, Flir Systems, Frankfurt am Main, Germany), fixed at a distance that allowed imaging the diffuser and the connector at the same time. Images were taken at a defined interval. Starting at room temperature, we determined the maximum temperature during the entire measured time period, and the mean temperature of the final record of the selected areas. It was assumed that the energy directly emitted by the sample had no influence on the measurement, since the spectral measurement range of the thermographic camera was between 10 and $14 \mu \mathrm{m}$, whereas the excitation of the laser-induced light is at $652 \mathrm{~nm}$.

\section{Results}

\subsection{Flexibility}

The forces to bend the samples through angles of 5 and $15 \mathrm{deg}$ (front and back) are shown in Fig. 3. We found all samples to be more flexible at the lower bending angle. In comparison to the optical lighting film, the textile diffusers were similarly easy to bend through $5 \mathrm{deg}$ lengthwise and $15 \mathrm{deg}$ crosswise, and easier to bend through $5 \mathrm{deg}$ crosswise and $15 \mathrm{deg}$ lengthwise. The results at the 15-deg bending angle (lengthwise and crosswise) for the textile diffusers show the highest variations, because stronger bending increases the effect of the easy-to-bend distances between the luminous elements, and the strong-to-bend POF, which both varied among the samples.

\subsection{Irradiance}

The irradiance measurement results are summarized in Table 1. The power of $100 \mathrm{~mW}$ input to the textile diffuser generated an average output power of $3.6 \pm 0.6 \mathrm{~mW} / \mathrm{cm}^{2}$ on the textile surface. Converted to the total luminous surface of the textile diffuser $\left(11.3 \mathrm{~cm}^{2}\right)$, this corresponds to an irradiance of $40.6 \mathrm{~mW}$. 
Table 1 Irradiance of seven textile diffusers, showing mean and standard deviation (SD) of 18 measurement points on each textile diffuser. Input power: $100 \mathrm{~mW}$.

\begin{tabular}{lcc}
\hline $\begin{array}{l}\text { Textile } \\
\text { diffuser } \\
\text { number }\end{array}$ & $\begin{array}{c}\text { Irradiance } \\
\text { mean } \\
(\mathrm{mW} / \mathrm{cm} 2)\end{array}$ & $\begin{array}{c}\text { Irradiance } \\
\text { SD } \\
(\mathrm{mW} / \mathrm{cm} 2)\end{array}$ \\
\hline 2 & 3.9 & 1.1 \\
8 & 3.3 & 0.8 \\
9 & 3.6 & 0.5 \\
10 & 3.9 & 0.5 \\
11 & 3.4 & 0.5 \\
12 & 3.1 & 0.4 \\
13 & 3.9 & 0.3 \\
Average & 3.6 & \\
\hline
\end{tabular}

The two tested backings indicated an average yield of $24 \%$ higher irradiance using the aluminum backing $\left(3.1 \pm 1.1 \mathrm{~mW} / \mathrm{m}^{2}\right)$ relative to the black backing $\left(2.5 \pm 0.7 \mathrm{~mW} / \mathrm{cm}^{2}\right)$.

\subsection{Brightness Distribution}

The brightness of the pixels vary in the range of millimeters [Fig. 4(a)]. The mean values of the distribution of dark and bright pixels of the six textile diffusers measured using the CCD camera are listed in Table 2. On a brightness scale of 0 to 255 , the peak in the distribution is at 65 on average, the median (Q50) averaged at 69, and the 95\% quantile (Q95) averaged at 124 (standard deviations are 7.3, 8.5, and 8.5, respectively). The average full-width at half maximum (FWHM) is 21 with a standard deviation of 4.6. The median (Q50) lies close at the peak, but constantly higher.

Table 2 Intensity level of maximum, 50\% quantile (Q50), full width at half maximum (FWHM), and 95\% quantile (Q95) of the brightness distribution histogram of the circular center area (brightness scale 0 to 255) of six textile diffusers.

\begin{tabular}{lcccc}
\hline $\begin{array}{l}\text { Diffuser } \\
\text { number }\end{array}$ & $\begin{array}{c}\text { Intensity } \\
\text { level }\end{array}$ & Q50 & FWHM & Q95 \\
\hline 2 & 69 & 75 & 26 & 135 \\
4 & 55 & 57 & 24 & 116 \\
9 & 69 & 75 & 24 & 130 \\
10 & 61 & 64 & 16 & 117 \\
11 & 61 & 63 & 15 & 116 \\
13 & 75 & 78 & 20 & 129 \\
Average & 65 & 69 & 21 & 124 \\
SD & 7.3 & 8.5 & 4.6 & 8.5 \\
\hline
\end{tabular}

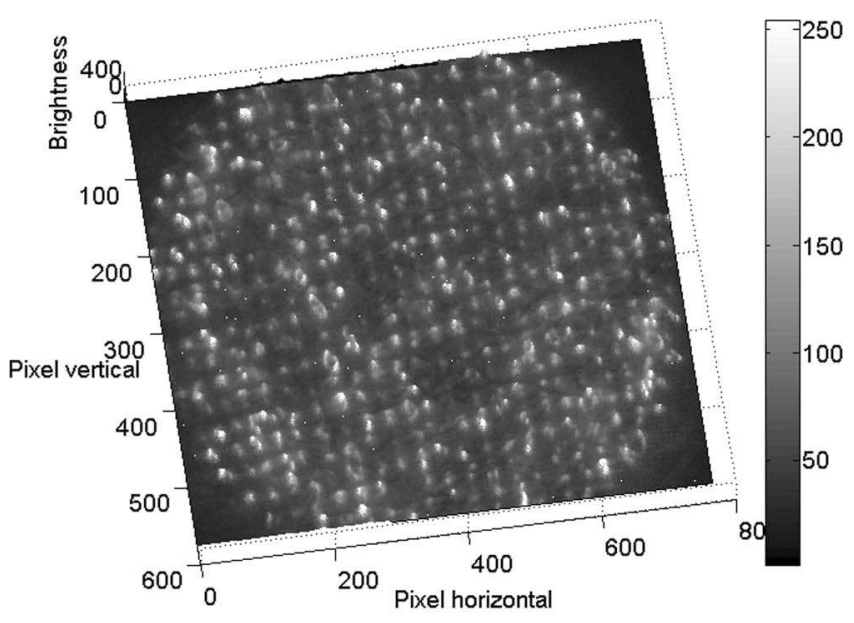

(a)

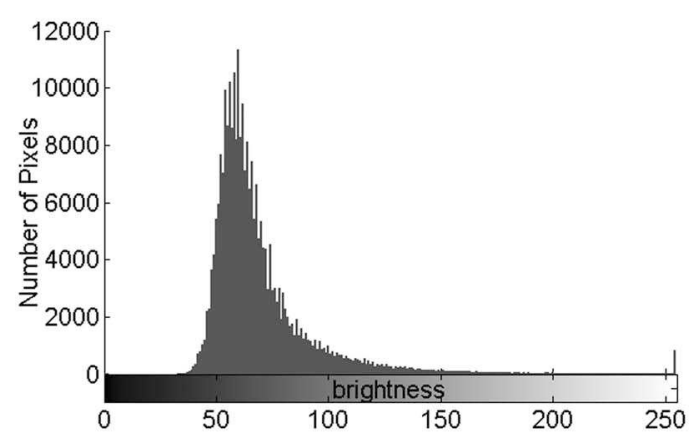

(b)

Fig. 4 (a) 3-D image and (b) brightness distribution (histogram) of the calculated luminous textile surface.

The skewed brightness distribution, as seen in Fig. 4(b), and for all textile diffusers in Table 2, shows a bell-shaped distribution with maximum and FWHM. The range from zero upward and the left steepness at 50 implies neither dark nor numerous faint pixels. At the other end of the scale, $5 \%$ of the pixels are at least twice as bright as the maximum level.

\subsection{Temperature}

In Table 3, the measured average temperature of each area at the end of several minutes of radiation and the maximum value over the entire specific period are listed. The results show clearly that, together with amplified radiation, the heat flux will also be increased. Power settings of $100 \mathrm{~mW}$ at the distal end of the frontal light distributor caused maximum temperature of $26^{\circ} \mathrm{C}$ at the measured areas on the textile diffuser. A power of $1 \mathrm{~W}$ heated the textile diffuser from $22^{\circ} \mathrm{C}$ to more than $30^{\circ} \mathrm{C}$ in less than $1 \mathrm{~min}$, and induced a mean temperature on the surface of $36^{\circ} \mathrm{C}$ and maximum values above $40^{\circ} \mathrm{C}$ after $5 \mathrm{~min}$.

The connector showed a lower heating rate. Adjusting power to $1.25 \mathrm{~W}$, the generated heat increase was almost $3 \mathrm{deg}$ (mean) and $10^{\circ} \mathrm{C}$ (maximum), respectively, within 5 min, reaching $25.8^{\circ} \mathrm{C}$ (mean) and $33.9^{\circ} \mathrm{C}$ (maximum), respectively, whereas on the textile surface, temperature approaches $40^{\circ} \mathrm{C}$ (mean) and $50^{\circ} \mathrm{C}$ (maximum), respectively. 
Table 3 Measured mean and maximum (max) temperatures of one textile diffuser (luminous area of $11 \mathrm{~cm}^{2}$, without aluminum foil) and connector, respectively, at the end of radiation for several minutes at different power settings. The irradiance of the textile diffuser surface was calculated.

\begin{tabular}{|c|c|c|c|c|c|c|}
\hline \multirow{3}{*}{$\begin{array}{l}\text { Power } \\
\text { settings } \\
(\mathrm{mW})\end{array}$} & \multirow{3}{*}{$\begin{array}{l}\text { Irradiance } \\
\text { (calculated) } \\
\text { (mW/cm2) }\end{array}$} & \multicolumn{4}{|c|}{ Temperature measurement $\left({ }^{\circ} \mathrm{C}\right)$} & \multirow{3}{*}{$\begin{array}{c}\text { Time } \\
\text { (minutes) }\end{array}$} \\
\hline & & \multicolumn{2}{|c|}{ Textile diffuser } & \multicolumn{2}{|c|}{ Connector } & \\
\hline & & Mean & Max & Mean & Max & \\
\hline 100 & 3.6 & 24.4 & 25.4 & 23.1 & 25.8 & 5 \\
\hline 100 & 3.6 & 25 & 26 & 23.5 & 26.2 & 10 \\
\hline 1000 & 36 & 32 & 39.5 & 23.1 & 25.4 & 1 \\
\hline 1000 & 36 & 35.2 & 43.1 & 24 & 29.5 & 5 \\
\hline 1000 & 36 & 35.7 & 43.7 & 24.5 & 31.4 & 7 \\
\hline 1250 & 45 & 38.4 & 48 & 25.8 & 33.9 & 5 \\
\hline
\end{tabular}

\section{Discussion}

Our aim was to develop a light applicator that would irradiate an area of almost any surface shape. With the present approach, it is possible to stitch the relatively brittle POF into arrays of intact, bent POF using an established embroidering method. Embroidery uses the flexible woven substrate, and with a small number of stitches, allows fixation of the POF, which in turn allows maximum flexibility in all directions of the textile diffuser, and therefore gives the textile haptic-a major advantage of the present development. Furthermore, our bending measurement results demonstrate low bending forces of the textile diffuser, i.e., high flexibility. The relatively high variation is likely due to the disordered bends and loops of the luminous fiber elements. However, compared with commercially available optical film, the textile diffuser is equally flexible or easier to bend.

In comparison to linear light diffusers, which in air preferentially radiate toward the cut off fiber end ${ }^{26}$ or laser beam profiles, which show microvariations of local irradiance ${ }^{27}$ but need some distance to reach the treatment surface, the present textile diffuser radiates randomly out of the surface. These results demonstrate that the emission of the required radiation power achieved on the textile diffuser is sufficient for PDT. These findings were confirmed by successful biological experiments using this kind of textile diffuser, ${ }^{28}$ and follow the trend to use low fluence rates for effective PDT. ${ }^{29}$ In our measurements, the textile diffuser achieved an irradiance of $3.6 \mathrm{~mW} / \mathrm{cm}^{2}$, corresponding to a mean efficiency of $40 \%$. According to our calculations, a maximum efficiency of $60 \%$ would be possible, whereas the maximum obtainable for many other systems is less than or equal to $60 \%$. $^{9}$ As expected, reflected radiation with the aluminum backing enhances the irradiance to the face (treatment side). The backing serves also as a reflecting coverage, which is useful to shield the treatment personnel. Furthermore, we observed steady light emission during bending of the textile diffuser by hand.
While the bend-induced light loss mechanism of POF is well known, ${ }^{24,30-33}$ the radiant exitance of complex structures like textiles is to the best of our knowledge underexplored. Connected to the laser, each of the stitched POF bends radiate in a unique manner. Our brightness distribution results indicate that most pixels show a specific homogeneous distribution, accomplished through a mixture of mainly scattered and redirected light. This multiple scattering saves space and allows irradiation of a full 2-D surface. The several percent $(5$ to $10 \%$ ) bright pixels, so-called hot spots, indicate powerful emission of the light mainly directed out of bent POF, and could result in overdosing during photodynamic treatment. As the scattering and absorbing properties of the treated tissue will also determine the light distribution in the tissue, ${ }^{26}$ the spatial variability of the textile diffuser emission on tissue needs to be better characterized, especially for the treatment of superficial and thin tissues. Nevertheless, a homogeneous 2-D irradiation of malignant cells is of essential importance for successful PDT. ${ }^{9}$ It was therefore an important confirmation of the present approach to find that the brightness distribution showed only a few isolated dark pixels.

It is difficult to compare the light distribution of the textile diffuser with previously reported systems, because not all relevant parameters are available. For comparison with the integrating balloon device, we did an approximate calculation on the uniformity of the irradiation as described by Dwyer et al., who analyzed images of PDT devices in a similar manner carried out here. Because the pixel brightness distribution of the textile diffuser is skewed, we employed the FWHM and Q50 (see Table 2) instead of the standard deviation and mean value, respectively. We found a slightly lower homogeneity of the textile diffusers (average uniformity $85 \%$, variation of standard deviation of $\pm 11 \%$ ) than that of the investigated balloon device of Dwyer's group (average uniformity of 93\%, standard deviation of $\pm 2 \%$ ). Several other groups used radiometric measurement setups to investigate the variations of irradiation of PDT devices. It is not clear that their results are directly comparable with the Dwyer algorithm. Nevertheless, these results seem to lie within a similar range and help to illustrate the state of the art. For laser beams (frontal light distributor), ${ }^{15,27}$ the percentage of variation of the light has been characterized as "very homogeneous" or shows variations up to $17 \%$. Variations of irradiance of different types of cylindrical light distributors (e.g., fiber optic, longitudinal balloon devices) ${ }^{10,15}$ can achieve very homogeneous results $( \pm 10 \%)$ along the main axis, and while showing good polar isotropy, the azimuth isotropy can vary by more than a factor of 5. The indwelling balloon applicator ${ }^{11}$ has been characterized as well to be "very homogeneous."

Regarding the potential for tissue warming at the illuminated areas of the skin, a combination with hemoglobin oxygenation $^{34}$ or hyperthermia ${ }^{35}$ was found. We measured the temperature increase of the isolated textile diffuser with a low irradiation dose $(100 \mathrm{~mW}$, associated with common PDT) over a period of $10 \mathrm{~min}$, and could show that heating would not be a concern for a potential patient nor for the device itself. We found that higher laser settings $(1 \mathrm{~W})$ could lead to temperatures over $37^{\circ} \mathrm{C}$ (normal body temperature is $37^{\circ} \mathrm{C}$ ) at the textile diffuser surface within a short time $(<5 \mathrm{~min})$. For higher laser settings in clinical practice, it is indispensable 
to use a good heat conductor like the aluminum backing, in good contact to the POF, to reduce temperature rise because the skin is a poor heat conductor.

\section{Conclusion}

The results of the radiation and homogeneity measurements presented here show that a textile diffuser using bent plastic optical fibers is feasible, and that the embroidered textile, together with an aluminum backing, supplies useful irradiation power for low-fluence-rate PDT treatment on an area of several $\mathrm{cm}^{2}$. In the future, the irradiation of uneven surfaces has to be investigated. Compared with existing irradiation systems, i.e., laser beams, frontal light distributors (microlens fiber), or balloon catheters, the new textile diffuser is thinner and more flexible and the variations of irradiance should be improved, especially for superficial applications.

Beyond these technical advantages, the present design offers easy handling, resembling an adhesive plaster, with a constant distance to the area to be treated. This allows considerable flexibility of movement (e.g., breathing), which is especially helpful for time-intensive PDT, as suggested by others, ${ }^{11}$ and avoids the need for complex energy computations.

The growing number of PDTs for dermatological cancer $^{36}$ suggests a parallel trend with applications with textile diffusers in the future. The textile diffuser was designed for applications to complex body surfaces and can also be used advantageously in body cavities for both nonmalignant disease and for cancer. Therefore the new flexible textile diffuser could revolutionize a number of well-established, effective PDT modalities $^{37}$ such as treatment of early dermato-oncologic stages like actinic keratoses, Bowen's disease, and superficial basal cell carcinoma. Besides these fields, the textile diffuser can offer potential advantages for PDT of body cavities prevalent in the head and neck area, such as for leukoplakia and more advanced stages of squamous cell carcinoma in the oral cavity.

\section{Acknowledgments}

The authors are grateful to the industrial partners Bischoff Textil AG, Saint Gallen, Switzerland, for numerous textile samples, and Biolitec AG, Jena, Germany for the borrowed laser equipment, numerous assembly operations, and helpful technical discussions. The authors thank the Interstate University of Applied Sciences of Technology Buchs NTB, Switzerland, for the cooperation resulting in the measurement setup. We acknowledge P. A. Brühwiler for the critical reading of the manuscript. The published work is supported by the Swiss Government Federal Office for Professional Education and Technology (BBT), Innovation Promotion Agency CTI, MedTech Initiative grant 6298.1 MTS.

\section{References}

1. J. A. Leman and C. A. Morton, "Photodynamic therapy: applications in dermatology," Expert Opin. Biolog. Therapy 2(1), 45-53 (2002).

2. I. J. MacDonald and T. J. Dougherty, "Basic principles of photodynamic therapy," J. Porphyr. Phthalocyanines 5(2), 105-129 (2001).

3. D. Dolmans, D. Fukumura, and R. K. Jain, "Photodynamic therapy for cancer," Nat. Rev. Cancer 3(5), 380-387 (2003).

4. J. S. McCaughan, "Photodynamic therapy-a review," Drugs Aging 15(1), 49-68 (1999)
5. T. J. Dougherty, "An update on photodynamic therapy applications," J. Clinic. Laser Med. Surg. 20(1), 3-7 (2002).

6. K. Plaetzer, T. Kiesslich, C. B. Oberdanner, and B. Krammer, "Apoptosis following photodynamic tumor therapy: Induction, mechanisms and detection," Curr. Pharmac. Design 11(9), 1151-1165 (2005)

7. R. M. Szeimies, R. Hein, W. Baumler, A. Heine, and M. Landthaler, "A possible new incoherent lamp for photodynamic treatment of superficial skin-lesions," Acta Derm Venereol 74(2), 117-119 (1994).

8. V. G. Schweitzer, "Photofrin-mediated photodynamic therapy for treatment of aggressive head and neck nonmelanomatous skin tumors in elderly patients," Laryngoscope 111(6), 1091-1098 (2001).

9. P. J. Dwyer, W. M. White, R. L. Fabian, and R. R. Anderson, "Optical integrating balloon device for photodynamic therapy," Lasers Surg. Med. 26(1), 58-66 (2000).

10. W. Beyer, "Systems for light application and dosimetry in photodynamic therapy," J. Photochem. Photobiol., B 36(2), 153-156 (1996).

11. S. J. Madsen, C. H. Sun, B. J. Tromberg, and H. Hirschberg, "Development of a novel in dwelling balloon applicator for optimizing light delivery in photodynamic therapy," Lasers Surg. Med. 29(5), 406412 (2001).

12. J. V. E. Roche, C. Whitehurst, P. Watt, J. V. Moore, and N. Krasner, "Photodynamic therapy (PDT) of gastrointestinal tumours: A new light delivery system," Lasers Med. Sci. 13(2), 137-142 (1998).

13. Y. Tadir, R. Hornung, T. H. Pham, and B. J. Tromberg, "Intrauterine lightprobe for photodynamic ablation therapy," Obstet. Gynecol. (N.Y., NY, U. S.) 93(2), 299-303 (1999).

14. R. L. P. van Veen, J. H. Schouwink, W. M. Star, H. Sterenborg, J. R. M. van der Sijp, F. A. Stewart, and P. Baas, "Wedge-shaped applicator for additional light delivery and dosimetry in the diaphragmal sinus during photodynamic therapy for malignant pleural mesothelioma," Phys. Med. Biol. 46(7), 1873-1883 (2001).

15. H. van den Bergh, "On the evolution of some endoscopic light delivery systems for photodynamic therapy," Endoscopy 30(4), 392-407 (1998).

16. R. Bays, G. Wagnieres, D. Robert, J. F. Theumann, A. Vitkin, J. F Savary, P. Monnier, and H. vandenBergh, "Three-dimensional optical phantom and its application in photodynamic therapy," Lasers Surg. Med. 21(3), 227-234 (1997).

17. L. M. Vesselov, W. Whittington, and L. Lilge, "Performance evaluation of cylindrical fiber optic light diffusers for biomedical applications," Lasers Surg. Med. 34(4), 348-351 (2004).

18. B. Selm and M. Camenzind, "Flexible textile light diffuser for photodynamic therapy," Proc. SPIE 5691, 95-103 (2005)

19. S. S. Hardaker and R. V. Gregory, "Progress toward dynamic colorresponsive 'chameleon' fiber systems," MRS Bull. 28(8), 564-567 (2003).

20. M. A. El-Sherif, J. M. Yuan, and A. MacDiarmid, "Fiber optic sensors and smart fabrics," J. Intell. Mater. Syst. Struct. 11(5), 407-414 (2000).

21. X. M. Tao, L. Q. Tang, W. C. Du, and C. L. Choy, "Internal strain measurement by fiber Bragg grating sensors in textile composites," Compos. Sci. Technol. 60(5), 657-669 (2000).

22. X. M. Tao, "Integration of fibre-optic sensors in smart textile composites: Design and fabrication," J. Text. Inst. 91(3), 448-459 (2000)

23. X. Tao, Wearable Electronics and Photonics, CRC Press, Boca Raton, FL (2005).

24. P. M. Pachomov, A. N. Zubkov, and S. D. Chiznjak, "Plastic filament as optical fiber," Chimiceskie Volokna 1, 46-50 (2000).

25. J. Zubia and J. Arrue, "Plastic optical fibers: An introduction to their technological processes and applications," Opt. Fiber Technol. 7(2), 101-140 (2001)

26. L. H. P. Murrer, J. P. A. Marijnissen, and W. M. Star, "Light distribution by linear diffusing sources for photodynamic therapy," Phys. Med. Biol. 41(6), 951-961 (1996).

27. T. J. Desmettre and S. R. Mordon, "Comparison of laser beam intensity profiles produced by photodynamic therapy (PDT) and transpupillary thermotherapy (TTT) lasers," Lasers Surg. Med. 36(4), 315322 (2005).

28. T. Khan, M. Unternährer, J. Buchholz, B. Kaser-Hotz, B. Selm, M. Rothmaier, and H. Walt, "Performance of a contact textile-based light diffuser for photodynamic therapy," Photodiag. Photodynam. Therapy 3(1), 51-60 (2006).

29. T. M. Sitnik, J. A. Hampton, and B. W. Henderson, "Reduction of tumour oxygenation during and after photodynamic therapy in vivo: 
effects of fluence rate," Br. J. Cancer 77(9), 1386-1394 (1998).

30. G. Durana, J. Zubia, J. Arrue, G. Aldabaldetreku, and J. Mateo, "Dependence of bending losses on cladding thickness in plastic optical fibers," Appl. Opt. 42(6), 997-1002 (2003).

31. J. Arrue, J. Zubia, G. Fuster, and D. Kalymnios, "Light power behaviour when bending plastic optical fibres," IEE Proc.: Optoelectron. 145(6), 313-318 (1998).

32. R. C. Gauthier and C. Ross, "Theoretical and experimental considerations for a single-mode fiber-optic bend-type sensor," Appl. Opt. 36(25), 6264-6273 (1997).

33. J. Zubia, J. Arrue, and A. Mendioroz, "Theoretical analysis of the torsion-induced optical effect in a plastic optical fiber," Opt. Fiber Technol. 3(2), 162-167 (1997).
34. A. A. Stratonnikov, N. V. Ermishova, and V. B. Loshchenov, "Diagnostics of a laser-induced response of capillary vessels in tissues," Quantum Electron. 32(10), 917-922 (2002).

35. J. Eichler, J. Liebetruth, R. A. London, and L. Ziegenhagen, "Temperature distribution for combined laser hyperthermia-photodynamic therapy in the esophagus," Med. Eng. Phys. 22(4), 307-312 (2000).

36. S. B. Brown, E. A. Brown, and I. Walker, "The present and future role of photodynamic therapy in cancer treatment," Lancet Oncol. 5(8), 497-508 (2004).

37. P. Babilas, S. Karrer, A. Sidoroff, M. Landthaler, and R. M. Szeimies, "Photodynamic therapy in dermatology—an update," Photodermatol. Photoimmunol. Photomed. 21(3), 142-149 (2005). 